\title{
Self Assembly and Optical Properties of Dendrimer Nanocomposite Multilayers
}

\author{
Lajos P. Balogh,* Shawn M. Redmond, Peter Balogh, Houxiang Tang, \\ David C. Martin, Stephen C. Rand
}

Ultrathin multilayers are important for electrical and optical devices, as well as for immunoassays, artificial organs, and for controlling surface properties. The construction of ultrathin multilayer films by electrostatic layer-by-layer deposition proved to be a popular and successful method to create films with a range of electrical, optical, and biological properties. Dendrimer nanocomposites (DNCs) form highly uniform hybrid (inorganic-organic) nanoparticles with controlled composition and architecture. In this work, the fabrication, characterization, and optical properties of ultrathin dendrimer/poly(styrene sulfonate) (PSS) and silver-DNC/PSS nanocomposite multilayers using layer-by-layer (LbL) electrostatic assembly techniques are described. UV-vis spectra of the multilayers were found to be a combination of electronic transitions of the surface plasmon peaks, and the regular frequency modulations attributable to the multilayered film structure. The modulations appeared as the consequence of the highly regular and non-intermixed multilayer growth as a function of the resulting structure. A simple model to explain the experimental data is presented. Use of DNCs in multilayers results in abrupt, flat, and uniform interfaces.

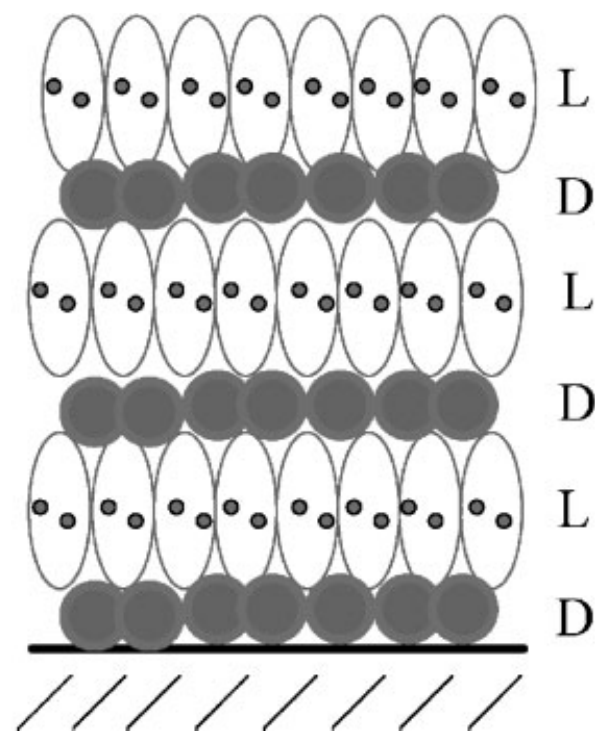

\section{Introduction}

Nanostructured clusters of semiconductors and metals possess unique properties as a result of electron confine-

L. P. Balogh

NanoBiotechnology Center at RPCI, Department of Radiation Medicine, Roswell Park Cancer Institute, Elm and Carlton Streets, Buffalo, NY 14263, USA

Fax: 716-845-8254; E-mail: lajos.balogh@roswellpark.org

S. M. Redmond, S. C. Rand

Department of Electrical Engineering and Computer Science,

University of Michigan, Ann Arbor, MI 48109-2122, USA

H. Tang, D. C. Martin, P. Balogh

Department of Materials Science and Engineering, University of

Michigan, Ann Arbor, MI, 48109-2106, USA ment. ${ }^{[1]}$ The unique properties of nanosized metal particles can be utilized in a broad range of fields, from catalysis to optical filters as well as non-linear optical devices. ${ }^{[2]}$

Ultrathin multilayers formed by the alternated adsorption of anionic and cationic polyelectrolytes on solid surfaces have proven to constitute an excellent way for the synthesis of multicomposite functional films of tailored architecture. $^{[3]}$ The concept of electrostatically driven assembly of multilayer structures allows for the incorporation of a wealth of different materials with varying film architectures. $^{[4-6]}$ Because branching polymers are typically flexible molecules, the resulting architectures generally lack crystallinity, ${ }^{[7]}$ and the individual layers may considerably penetrate into each other. 
In spite of achievements, there are several limitations for linear ionic polymers, such as broad polydispersity, difficult coupling with other molecules, lack of steady size and shape, long deposition times because of the low critical micelle concentration, etc. Ideally, polyelectrolyte multilayers have a 1:1 stoichiometry of anionic and cationic groups as every anionic group of a polyanion is bound to a cationic group of a polycation. This is also the case in bulk polyion complexes of flexible polyelectrolytes of high charge density and similar molecular weights. ${ }^{[8]}$

For weak polyelectrolytes, not all of the monomers need to be charged, so that the overall stoichiometry may deviate from 1:1. ${ }^{[9]}$ This has the consequence that the concentration of anionic and cationic groups must be identical throughout the polyelectrolyte multilayer film and constant along the layer normal. Such a homogenous distribution of charged ionic groups in the film do not contradict the notion of defined individual layers of polyions within such multilayer assemblies. ${ }^{[10]}$

Numerous studies have been devoted to understanding the buildup of these multilayers. In general they are simply formed by an alternate dipping of the supporting surface into solutions of anionic and cationic polyelectrolytes, the layers being afterward rinsed with pure water or buffer and dried with nitrogen before being again immersed into the solution. The multilayer thickness seems then to increase linearly with the number of constituting pairs of polyanion/polycation. The number of pairs that can be adsorbed seems almost infinite so that very large film thicknesses are attainable. ${ }^{[1]}$

More precisely, the basic structure of a multilayer film is subdivided into three zones. ${ }^{[4]}$ The first layers, deposited close to the substrate, will be governed by the interference with the substrate. Typically the thickness per layer in Zone I is slightly smaller than that in Zone II. ${ }^{[12]}$ This Zone I is typically composed of only a few layers. In Zone II, in the 'bulk' multilayer film, all anionic layers and all cationic layers possess equal thickness. In most cases the polyanion/polycation stoichiometry is observed to be 1:1 or at least close to that value. Zone III is the region close to the surface of the film. This region is typically composed of only a few layers, and it could be described as a transition zone between the charge-compensated region II and the charged surface. The transitions between Zones I and II and between II and III are gradual. While Zone II should be zwitterionic in nature, the layers in Zone III should not be charge compensated and thus show classic polyion-like behavior. When the film is fabricated, Zone I is completed first. As more layers are added, Zones I and III will preserve their respective thicknesses while Zone II will grow in thickness.

In our work poly(amidoamine) (PAMAM) dendrimer ${ }^{[13]}$ templates were used to prepare multilayers of dendrimers, silver-dendrimer complexes, and silver-dendrimer nano- composites on different substrates using layer-by-layer assembly techniques.

\section{Dendrimers}

Dendrimers $^{[14-16]}$ are nearly monodisperse macromolecules that contain symmetric branching units built around a small molecule or a linear polymer core. The high level of synthetic control makes possible the synthesis of a narrow molecular weight range of well-defined and highly symmetrical polymer molecules, which contain a large number of regularly spaced internal and external functional groups. However, 'dendrimer' is only an architectural motif and not a compound. Polyionic dendrimers do not have a persistent shape, and may undergo changes ${ }^{[17]}$ in size, shape, and flexibility as a function of increasing generations. $^{[18-20]}$

\section{Dendrimers on Solid Surfaces}

Dendrimers readily interact with solid surfaces because of their numerous terminal groups and form either monolayer or multilayer structures on various substrates. ${ }^{[21]}$ Composite films of $(\mathrm{AB})_{x}$ type were fabricated by the self-assembly of PAMAM dendrimers with surface amine groups and carboxylic groups of two adjacent generations using electrostatic layer-by-layer deposition. ${ }^{[2]}$ All even generations were observed to form homogeneous, stable monolayers on a silicon surface. However, in these films the PAMAMs were collapsed and highly compressed along the surface normal, which resulted in flattened, disk-like structures. The average thickness of a single layer in multilayer films was much smaller than the diameter of the ideal spherical dendritic macromolecule in solution. The model of dendrimer films assumed compressed dendritic macromolecules of oblate shape with an axial ratio in the range from 1:3 to 1:6. This deformation had been predicted by applying a diamond lattice dendrimer model ${ }^{[23]}$ without using any composition-related information.

The high interaction strength between the large number of surface groups along with short-range van der Waals forces and long-range capillary forces are considered to be responsible for the formation of these compact monolayer structures. The observed deformation was explained by strong electrostatic interactions between the terminal cationic functional groups and the negatively charged substrate. ${ }^{[24]}$

Apparently, the size of the flattened structures also depends on the water content of the layers formed. The thickness of a single monolayer varied with generation and was found to be $1.8 \mathrm{~nm}$ for generation 4 and $2.8 \mathrm{~nm}$ for generation 6 PAMAM. ${ }^{[25,26]}$ However, when multilayers of 
PAMAM_E6. $\mathrm{NH}_{2}$, and PAMAM_E4. $\mathrm{NH}_{2}$ dendrimers have been grown on a silicon wafer by sequential deposition ${ }^{[27]}$ onto a $\mathrm{Pt}^{2+}$-bearing surface, the ellipsometric measurements indicated an average thickness per layer of 8.0 and $5.0 \mathrm{~nm}$, respectively, which values were in reasonable agreement with estimated diameters for these dendrimers in solution. Examination by atomic force microscopy (AFM) revealed that the surface was very smooth at the macromolecular level, and the average roughness was $7.1 \AA$.

Using poly(styrene sulfonate) (PSS) as the interlayer, Caruso and co-workers assembled PAMAM multilayers onto a planar surface using PAMAM_E2. $\mathrm{NH}_{2}$, PAMAM_E3. $\mathrm{NH}_{2}$, and PAMAM_E4. $\mathrm{NH}_{2}$ dendrimers and low-molecular-mass $\left(\bar{M}_{\mathrm{n}}=13 \mathrm{k}-70 \mathrm{k}\right)$ PSS. ${ }^{[28,29]}$ When PAMAMs with different generation numbers were used to deposit multiple layers, the PSS removal profile showed divergence, convergence, and sigmoidal behavior, which were dependent on the PSS/dendrimer charge ratio. It was concluded that during the formation of the layers, partial removal of PSS from the film may also occur.

This phenomenon, i.e., that the top layer of adsorbed material is partially removed by the solution (either the polycationic top layer by the PSS solution, or the PSS top layer by the dendrimer solution) clearly highlighted the importance of a standardized experimental technique, in which short contact times are applied and the removal of unbound layers are optimized.

\section{Dendrimer Nanocomposites}

Dendrimer nanocomposites $(\mathrm{DNCS})^{[30-32]}$ are recently developed materials ${ }^{[3,34]}$ made by reactive encapsulation and composed of nanoscopic inorganic guest domains and a dendritic polymer host and contain no covalent bonds between host and guest(s). In this reactive encapsulation method $^{[32]}$ dendrimers are used as templates to preorganize small molecules or metal ions followed by an in-situ immobilization of atomic or molecular domains of various inorganic guests. The procedure provides excellent control over size and size distribution of the hybrid nanoparticles. Interactions of DNCs with the molecular environment (including solubility) are determined by the host polymer molecules, however, they also possess many of the desirable chemical and physical properties of the guest molecules or atoms. DNCs often display unique physical and chemical properties ${ }^{[35-38]}$ as a consequence of the atomic/molecular level dispersion of their components.

\section{DNC Films}

We have reported ${ }^{[39]}$ a quick and efficient strategy to engineer nanostructured multilayer films with variable composition, controlled thickness, and well-defined reg- ular architecture. This approach has combined the layerby-layer technique with the concept of dendrimer and DNC synthesis for quick production of uniform heterostructured films. The combination of the plasma treatment of the substrate ${ }^{[40]}$ with the layer-by-layer electrostatic self-assembly of a linear polymer and a gold DNC opened a new approach for constructing controlled inorganic/ organic nanostructured films. This technique is completely different from those when dendrimers are used as a stabilizer for preformed silver colloids, ${ }^{[41,42]}$ in which case the high absorption tendency of dendrimer terminal groups is used to 'glue' nanoparticles together in an amorphous film.

An intriguing observation for $\left\{\mathrm{Au}^{\circ}\right\} /$ SPS gold-PAMAM nanocomposite multilayers was ${ }^{[39]}$ that although the absorbance of the deposited gold-DNC in the UV region increased with the number of bilayers, the plasma peak at $529 \mathrm{~nm}$ did not appear until six adsorption cycles, even though a film with a golden hue was easily observable from the substrate to the naked eye. A possible explanation for the absence of the plasma peak was that the ultrathin film (less than 8 bilayers) of the PSS/gold-DNC structure was highly smooth and acted like a gold mirror to reflect the incident light and mask the plasma absorption peak. Upon repeating the dipping cycle, both the thickness and the surface roughness of the film increased and the reflection and the mirroring effect was gradually lost after the deposition of eight bilayers, and only the characteristic peak of the gold particles was observed.

One of our objectives from the start was to find out whether this phenomenon existed in other DNC multilayer systems.

\section{Silver-DNCs}

Silver-PAMAM DNCs are soft nanosized polymeric particles that contain dispersed silver ions/atoms and/or small clusters separated by the dendrimer wedges. The synthesis of silver dendrimer complexes and nanocomposites has been reported both by direct complexation ${ }^{[43]}$ and by substitution of copper in the preformed copper nanocomposite. ${ }^{[4]}$ According to X-ray and neutron diffraction measurements, ${ }^{[31,45,46]}$ the size of the dendrimers is usually not altered by the metal ion complexation.

The formation mechanism of silver DNCs using PAMAM dendrimers has been studied in detail. ${ }^{[4,47-50]}$ These silver-dendrimer complexes and nanocomposites are stable and soluble in polar solvents, such as water, methanol, etc. In preliminary experiments the solutions of silver-PAMAM complexes proved to be quite insensitive to light: one day exposure to daylight resulted in only approximately $50 \%$ conversion based on increase of intensity of the $420 \mathrm{~nm}$ surface plasmon (SP) peak in the visible spectra. 
Photolysis of complexed silver ions generated metallic silver domains. ${ }^{[4,46,48]}$ Location of the silver domains depends on the metal-to-dendrimer ratio, the structure of the template, and on the kinetics of the immobilizing reaction. It was originally assumed that when nanocomposite formation is a zero-order process, metal atoms form at the same location where the silver ions were originally bound to the dendrimer structure. This was suggested by the UV-visible spectra, which displayed only one major symmetric plasmon peak at $\lambda 420 \mathrm{~nm}$ in both nanomaterials. ${ }^{[43]}$ A more detailed investigation based on electron paramagnetic resonance (EPR) techniques ${ }^{[48]}$ concluded that in PAMAM complexes the silver ions are bound to the nitrogen ligands while neutral silver atoms in the nanocomposites occupy the smaller cavities of the dendrimers.

Electrostatic self-assembly of DNCs opens up the possibility to construct multilayers of polymer-protected nanometer-scale metal domains with a range of interesting physical and biological properties. For example, non-linear optical behavior ${ }^{[47]}$ and large optical limiting ${ }^{[51]}$ (by factors up to 115 at $532 \mathrm{~nm}$ ) was found for a solution of $\left\{\mathrm{Ag}^{0}\right\}_{\mathrm{E}}$ external type DNCs. Laser-induced optical breakdown was also observed, which was later studied by acoustic methods. ${ }^{[52,53]}$

Dendrimer-silver complexes and nanocomposites also exhibit interesting antimicrobial activity. ${ }^{[54]}$

\section{Experimental Part}

\section{Materials}

Quartzglass SUPRASIL 665.000-OX polished quartz slides (45 $\times$ $12.5 \times 1.25 \mathrm{~mm}^{3}, n_{i}=1.458$ ) were used as a substrate. Multilayers were also constructed on freshly cleaved mica sheets (montmorillonite, $40 \times 9.5 \mathrm{~mm}^{2}$ ). For transmission electron microscopy (TEM) cross-section images a polystyrene cover slip (Fischer) was used which had a thickness of $1 \mathrm{~mm}$.

High-molecular-weight sodium PSS was purchased from Dajac Laboratories, Inc., and it had an average molecular weight of $5000000 \mathrm{Da}$. It was used at a concentration of $0.0991 \mathrm{~g}$ per $100 \mathrm{~mL}$ Dendrimers were purchased from Dendritech, MI and were used without further purification. Silver acetate (99.999\%) was purchased from Aldrich and was stored in an exsiccator. Concentrations of dendrimer solutions were kept constant at about $0.10 \mathrm{~g}$ per $100 \mathrm{~mL}$ (Table 1). The metal-to-dendrimer ratio is predetermined by the ratio of metal ion moles per dendrimer moles because of the uniformity of dendrimers and the isotropic nature of the diffusion. Accordingly, individual dendrimers form complexes with an equal and well-defined number of metal atoms per dendrimer, the ratios of which are expressed as average numbers per dendrimer molecules.

\section{Techniques}

Starting materials and the obtained products were carefully characterized by different analytical techniques. UV-visible spectra were obtained on a Perkin Elmer Lambda 20 spectrophotometer at room temperature between 200 and $1100 \mathrm{~nm}$ in a Suprasil 300 quartz cell $(L=1 \mathrm{~mm}) .{ }^{1} \mathrm{H}$ and ${ }^{13} \mathrm{C}$ NMR measurements of the dendrimers were carried out on a Bruker 500 multinuclear spectrometer equipped with a temperature controller. Size exclusion chromatography (SEC) was performed on three TSK gel columns (4 000, 3000 , and 2000) using a Waters 510 pump with a Wyatt Technology Dawn DSP-F MALLS and Wyatt Technology 903 interferometric refractometer and a Waters 510 pump with a Waters 410 differential refractometer, respectively.

TEM samples were prepared from the $\left.\left(\left\{\mathrm{Ag}_{66}-\mathrm{E} 5\right)\right\} / \mathrm{PSS}\right)_{24}$ multilayers deposited on a polystyrene slide. Crossections of the multilayer films were imaged. The polystyrene coated by the $\left.\left(\left\{\mathrm{Ag}_{66}{ }^{-\mathrm{E} 5}\right)\right\} / \mathrm{PSS}\right)_{24}$ multilayers was microtomed with a 45 degree diamond knife on a Reichert-Jung Ultracut E microtome. The thickness of the sample was about $70 \mathrm{~nm}$. The sample was then coated with a thin layer of carbon before it was observed on a Jeol $4000 \mathrm{EX}$ TEM operating at $400 \mathrm{kV}$.

\section{Preparation of Silver-Dendrimer Complexes and Nanocomposites ${ }^{a}$}

\section{Complexation}

For complexation experiments, silver acetate was selected because it is practically insoluble in water except for its dendrimer complex. To reflect the contribution of architectural differences among various dendrimer templates and nanocomposite structures two sets with different variables were prepared. The variance in measured characteristics, therefore, reflects dominantly the composition and architecture of the nanoparticles used. In experiments A, $\mathrm{C}$, and $\mathrm{E}$ the ratio of $\mathrm{Ag} / \mathrm{N}$ i.e., silver/amine nitrogens was kept constant. In this set, macromolecules with increasing size had the same relative number of silver ions per dendrimer. In experiments $B, C$, and D, the same generation five $(d=5 \mathrm{~nm})$ dendrimer was used but the relative concentrations were different.

\footnotetext{
a We use the following convention to describe the composition of the synthesized nanomaterials: brackets denote complexes while braces represent disperse nanocomposite structures. Within the brackets or braces first the encapsulated components are listed then the family of dendrimers follows by terms used for dendrimer identification, i.e., core, generation and surface. E.g.:\{(compo-

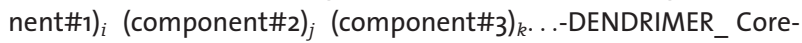
Generation.Terminal group $\}$. Thus, the formula of $\left\{\left(\mathrm{Ag}^{\circ}\right)_{17}-\mathrm{PAMAM}\right.$ $\mathrm{E} 5 . \mathrm{NH}_{2}$ \} denotes a silver dendrimer nanocomposite in which a generation five ethylenediamine core poly(amidoamine) (PAMAM) dendrimer $\left(M_{\mathrm{w}}=28826\right)$ with amine terminal groups that contains seventeen zerovalent silver atoms $\left(\left\{\mathrm{Ag}_{17} \mathrm{E} 4\right\}\right.$ for short in this paper, as all dendrimers used here were primary amine terminated ones) and $\left[\left(\mathrm{Ag}^{+}\right)_{66}\right.$-PAMAM_E6.NH $\left.{ }_{2}\right]$ denotes the silver complex of an EDA core generation six PAMAM complexing 66 silver ions per dendrimer on average ( $\left[\mathrm{Ag}_{66} \mathrm{E} 6\right]$ for short in this paper). Accordingly, $\left(\left\{\mathrm{Ag}_{33} \mathrm{E}_{5}\right\}\right)$ PSS $)_{24}$ identifies a multilayer structure consisting of 24 bilayers on both sides of a substrate in which every bilayer was constructed by a sequential deposition of an $\left\{\mathrm{Ag}_{33} \mathrm{E}_{5}\right\}$ nanocomposite followed by a PSS layer.
} 
Table 1. Data of silver-dendrimer complexes and nanocomposites.

\begin{tabular}{|c|c|c|c|c|c|c|c|c|c|}
\hline \multirow{2}{*}{$\begin{array}{l}\text { Exp. } \\
\text { No. }\end{array}$} & \multirow{2}{*}{ Dendrimer } & \multirow{2}{*}{$\frac{\text { Diameter }}{\mathrm{nm}}$} & \multirow[t]{2}{*}{$\mathrm{F}_{\mathrm{w}}{ }^{\mathrm{a})}$} & \multirow{2}{*}{$\begin{array}{c}\text { No. of } \\
\text { primary } \\
\text { amines }\end{array}$} & \multirow{2}{*}{$\begin{array}{c}\text { No. of } \\
\text { tertiary } \\
\text { amines }\end{array}$} & \multirow{2}{*}{$\begin{array}{l}\text { Total no. } \\
\text { of nitrogens }\end{array}$} & \multirow{2}{*}{$\frac{\mathrm{CH}_{3} \mathrm{COOAg}}{\mathrm{g} \cdot \mathrm{L}^{-1}}$} & \multirow{2}{*}{$\frac{[\mathrm{D}]\left(\times 10^{-6}\right)}{\mathrm{mol} \cdot \mathrm{L}^{-1}}$} & \multirow[t]{2}{*}{$\mathrm{Ag} / \mathrm{D}$ ratic } \\
\hline & & & & & & & & & \\
\hline A & E4.A & 4.5 & 14215 & 64 & 62 & 126 & 0.210 & 1.93 & 17.47 \\
\hline B & E5.A & 5.4 & 28826 & 128 & 126 & 254 & 0.210 & 4.44 & 16.58 \\
\hline C & E5.A & 5.4 & 28826 & 128 & 126 & 254 & 0.216 & 3.75 & 33.54 \\
\hline $\mathrm{D}$ & E5.A & 5.4 & 28826 & 128 & 126 & 254 & 0.123 & 3.78 & 65.27 \\
\hline E & E6.A & 6.7 & 58048 & 256 & 254 & 510 & 0.412 & 1.93 & 66.94 \\
\hline
\end{tabular}

a) Formula weight.

Multilayer Preparation

Quartz slides were cleaned using a Fischer Scientific FS6 ultrasound cleaner. The slides were treated first in a Hellmanex II cleaning solution, and then in water. Slides were soaked three times for eight hours in a deionized water bath, in which the water was changed. Prior to deposition, the slides were dried and scoured with a PDC-3xG plasma cleaner (Harrick Scientific).

Ultrathin films were formed by using a slightly modified version $^{[39]}$ of the procedure described in the literature. ${ }^{[3,4]}$ First, the substrates were immersed into dilute $(0.1 \%)$ solutions that contained PAMAM (dendrimers or silver complexes). After one minute the slides were rinsed with deionized water and then gently dried with nitrogen gas, prior to a second immersion for five minutes in a $0.1 \%$ PSS solution. A second rinse/dry cycle completed the formation of a single 'bilayer' on each side of the quartz substrate surface. This process was repeated until 24 bilayers were formed on each side. The absorption characteristics of the film samples at deposition increments of one to three bilayers per side were measured using a Perkin Elmer Lambda 20 UV/ visible spectrophotometer between 200-1000 nm. The sample data was calibrated to the absorption of PSS at 224 and $260 \mathrm{~nm}$ to estimate the amount of solid deposited on each quartz slide. For this procedure PSS solutions were measured in quartz cuvettes ( 0.100 and $1.000 \mathrm{~cm}$ thickness) using the same spectrophotometer. Molar extinction coefficients were assumed to be identical for PSS in solution and in the layers. (The same procedure cannot be applied to dendrimers and nanocomposites as their shape, volume, and density changes depending on their composition, generation, and the amount of solvent contained.) The amount of complexed silver was doubled from $B$ to $C$ and $C$ to $D$, respectively. The metal ion-to-dendrimer ratio is determined by the ratio of metal ion moles per dendrimer moles because of the uniformity of dendrimers and the isotropic nature of the diffusion in solution. Accordingly, individual dendrimers will form complexes with an equal and well-defined number of metal atoms per dendrimer molecule, which are expressed as average numbers (Table 1 ).

Exposure of the silver complex solutions to visible light over an extended time yielded yellow to light brown silver nanocomposite solutions, which did not precipitate even over a long period of time from their respective solutions. Silver nanocomposite stock solutions were stored at room temperature in sealed vials wrapped with several layers of aluminum foil. To prevent unwanted exposure to light during the multilayer fabrication, the deposition process was carried out under low light conditions. Of course, exposure of the samples to light cannot be avoided in the spectrophotometer and exposure to the electron beam in the TEM completes the photochemical transformation.

\section{Results and Discussion}

\section{Transmission Features of Dendrimer and Nanoparticle Films}

After identifying that the same general pattern exists in silver nanocomposite multilayers as for gold nanocomposites, we studied its origin by relating the observed modulations to individual layer thicknesses with a contact-free method. Figure 1 shows the characteristic absorptions of the materials used.

To characterize the optical properties of the dendrimer and DNC thin film structures as a function of sample thickness, the absorption and transmission spectra of multilayers were recorded subsequent to the deposition of E5/PSS dendrimer/PSS (Figure 2), and $\left\{\mathrm{Ag}_{n}-\mathrm{Em}\right\} / \mathrm{PSS}$ nanocomposite/PSS multilayers (Figure $3(\mathrm{a})-(\mathrm{c})$ ). Once each

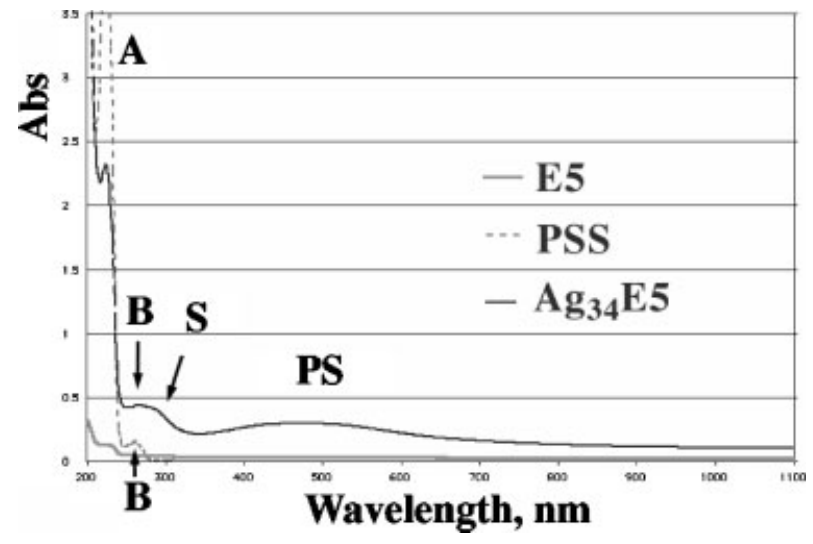

Figure 1. Comparison of respective solution spectra of PAMAM E5, $\left\{\mathrm{Ag}_{34} \mathrm{E}_{5}\right\}$ silver nanocomposite, and PSS. 

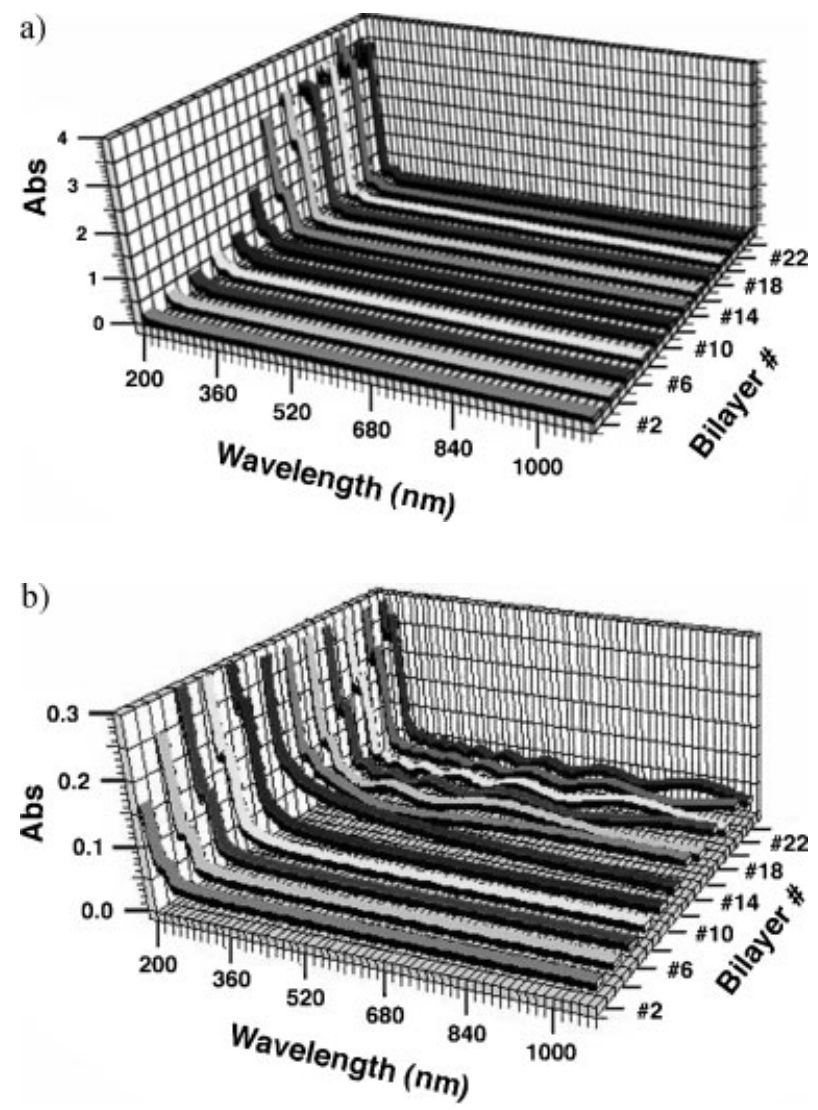

Figure 2. Consecutive absorption spectra of E5/PSS multilayers in the high (a) and in the low (b) absorbance regions as a function of the number of bilayers.

spectroscopic scan was completed, growth was resumed until a film structure of up to 24 bilayers was formed on each side of the substrate. Quartz, mica, and polystyrene were used as substrates.

The absorbance increased with increasing number of bilayers (Figure $4(\mathrm{a})-(\mathrm{c})$ ). Samples that consisted of less than nine bilayers revealed essentially featureless spectra. All the constituents of the multi-layer structure, including the substrate, were highly transparent at wavelengths longer than $200 \mathrm{~nm}$. Near the absorption edge, the coefficient of absorption was close to linear in the number of deposited layers.

Typical features observed in consecutive spectra of nanocomposite/PSS multilayers were:

- gradual increase of the peak at $\lambda=224 \mathrm{~nm}$ in the high intensity region $(A=0-3.5)$ which indicates the buildup of layers,

- modulations, which appeared in the multilayer UV-vis spectra in the low intensity region $(A=0-0.3)$ after a few bilayers have been deposited,

- growth of plasmon peaks, which are characteristic of composite nanoparticles (silver particles confined in a dendrimer).
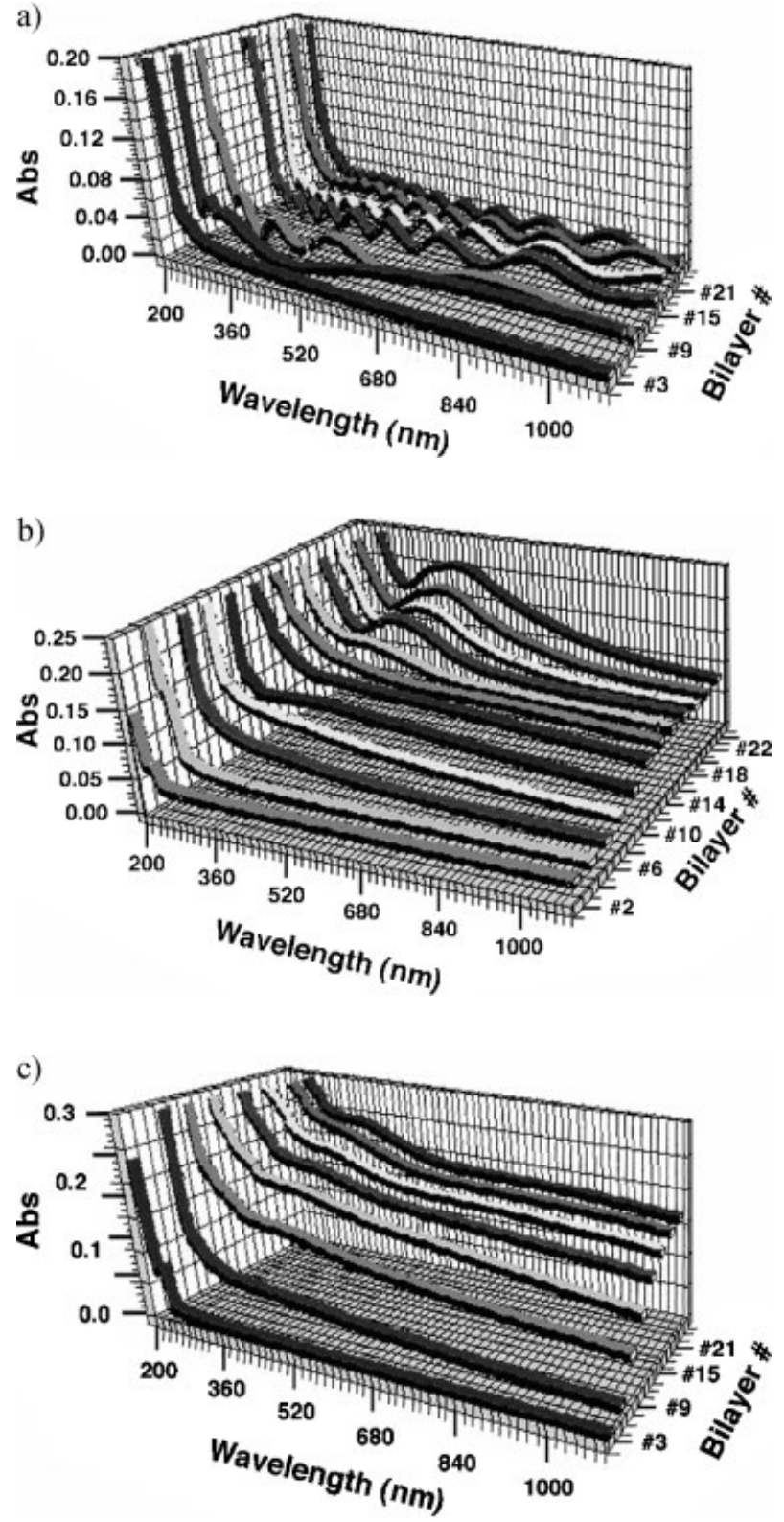

Figure 3. Consecutive absorption spectra of progressive multilayer deposition on quartz substrate for $\left\{\mathrm{Ag}_{17} \mathrm{E}_{5}\right\} / \mathrm{PSS}(\mathrm{a}),\left\{\mathrm{Ag}_{33} \mathrm{E}_{5}\right\}$ / PSS (b), and $\left\{\mathrm{Ag}_{66} \mathrm{E}_{5}\right\} /$ PSS (c).

All three phenomena are well illustrated in Figure 5 and 6.

The modulation frequency increased with increasing number of deposited bilayers, while its amplitude essentially remained the same or very similar within one experiment. The modulation frequency decreased with increasing silver concentration (Figure 3 and Figure 4). Plasmon peaks were fairly broad and had different center wavelengths. 

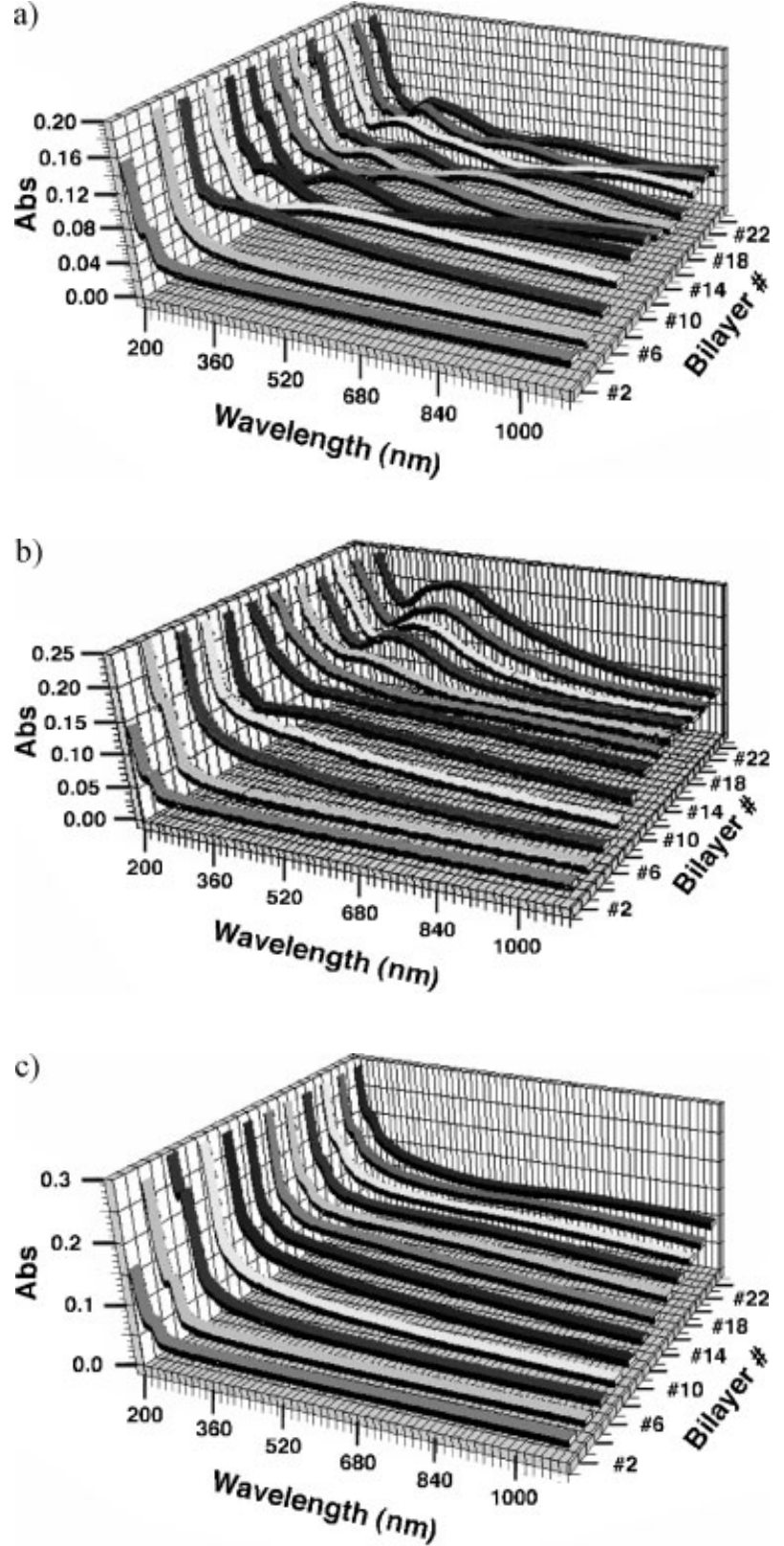

Figure 4. Consecutive absorption spectra of progressive multilayer deposition on quartz substrate for $\left\{\mathrm{Ag}_{17} \mathrm{E} 4\right\} / \mathrm{PSS}(\mathrm{a}),\left\{\mathrm{Ag}_{33} \mathrm{E} \mathrm{E}_{5}\right\}$ PSS (b), and $\left\{\mathrm{Ag}_{66} \mathrm{E} 6\right\}$ /PSS (c).

\section{Estimation of Layer Thicknesses Based on Calibration with PSS Solutions}

Both the polyanionic PSS and the PAMAM dendrimer absorb light at $\lambda=224 \mathrm{~nm}$. Calibration curves for these two peaks were prepared using ultraviolet scans of PSS solutions of known density and are given in Figure 7. Although the observed absorbance must be proportional to the weighted sums of the contributions of all layers, PAMAMs

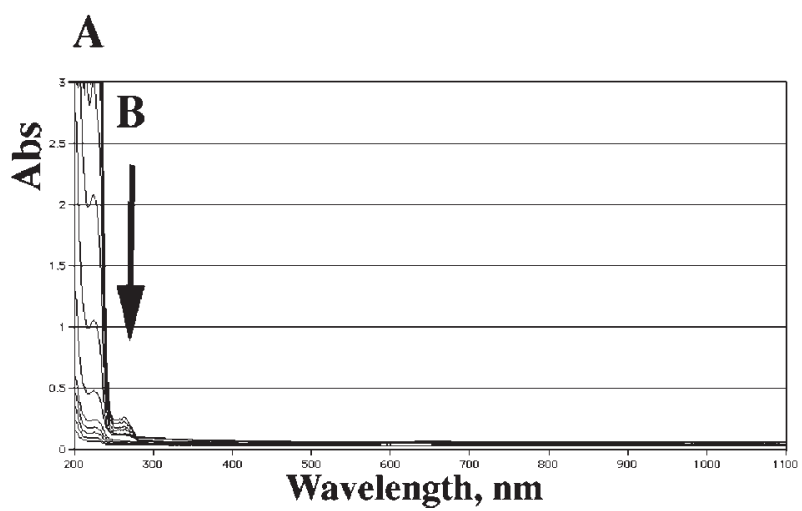

Figure 5. Consecutive UV-vis spectra of (E5/PSS) ${ }_{24}$ multilayers on quartz in the high intensity regime. PAMAM dendrimers have low UV-vis absorption, accordingly this multilayer spectrum is governed by the absorption of the increasing number of polystyrene layers. However, Peak A is the sum of PAMAM and PSS absorptions, while the $\lambda=254 \mathrm{~nm}$ peak, which is characteristic of aromatic rings, is dominated by the PSS absorption.

have negligible contributions at both wavelengths, ${ }^{[51]}$ and PSS calibrations are a good estimate.

\section{Effect of Substrate}

Three different substrates were used: quartz, mica, and polystyrene. Suprasil quartz windows have at least $85 \%$ transmission over the whole spectroscopic range. However, because of the fine layered structure of the montmorillonite, when mica was used as a substrate, secondary modulations show up on the lower frequency modulations caused by the deposited thin film structures (Figure 8).

We have also confirmed the structure of the nanocomposite multilayers by the visualization of the metal

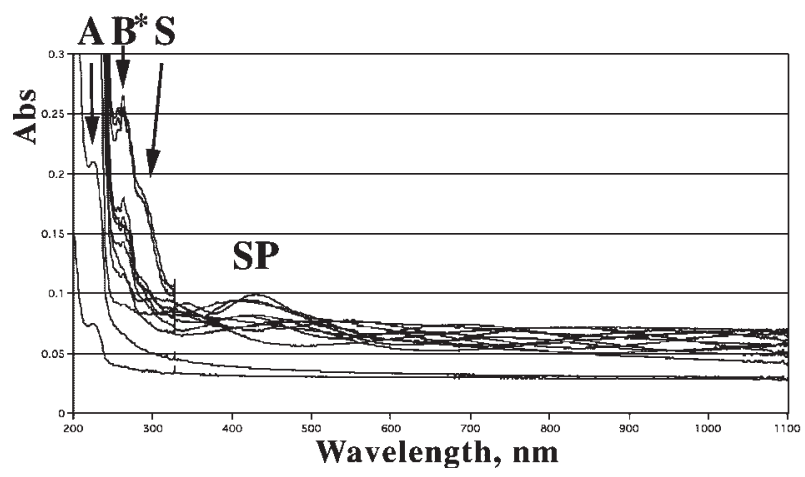

Figure 6. Consecutive spectra of $\left\{\mathrm{Ag}_{17} \mathrm{E} 4\right\} / \mathrm{PSS}$ multilayers on quartz in the low intensity regime. $A$ : polystyrene and PAMAM peak, $B^{*}=(B+S)$, i.e., polystyrene and the contribution of the overlapping part of the $S$ single nanocomposite absorptions at 290-300 nm, S: single nanocomposite peak (as a result of confined ions/atoms), SP surface plasmon peak caused by interacting silver domains. Regular oscillations could be observed at wavelengths longer than $\lambda=550 \mathrm{~nm}$. 


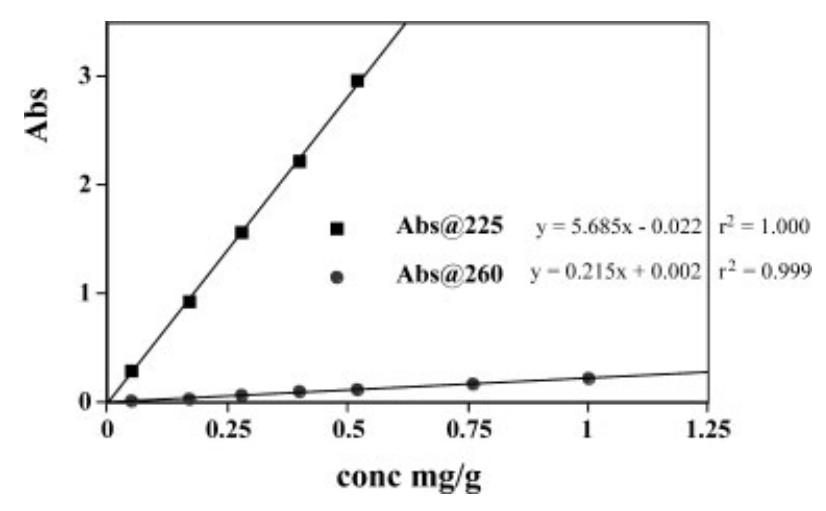

Figure 7. Standard calibration curves for PSS as measured in solution at peak maximum values, i.e., $\lambda=225$ and $260 \mathrm{~nm}$ wavelengths.

containing layers. In order to get good quality crosssections, polystyrene was used as a substrate for $\left\{\mathrm{Ag}_{66} \mathrm{E} 6\right\}$ / PSS multilayer deposition employing the same materials and procedure previously used for quartz. Use of a polystyrene substrate eliminates the possibility of observing any styrene or PAMAM-related spectroscopic changes,
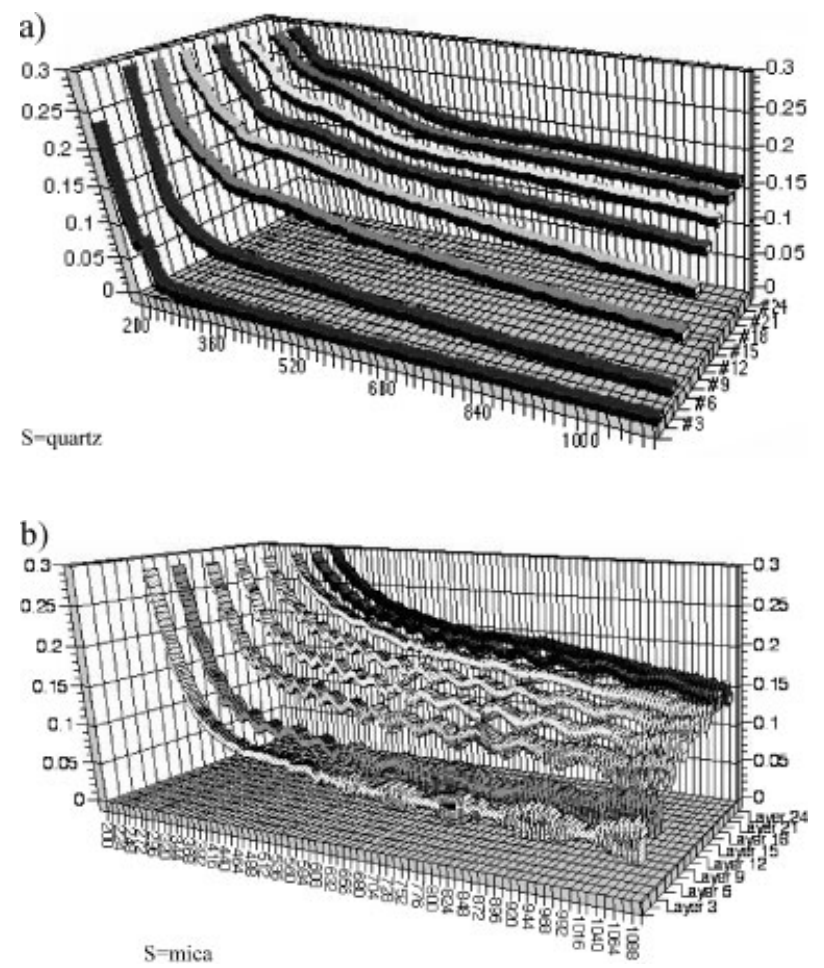

Figure 8. Consecutive spectra of $\left\{\mathrm{Ag}_{66} \mathrm{E}_{5}\right\} / \mathrm{PSS}$ multilayers on quartz (a) and mica (b). When quartz has been used as the substrate only weak modulations as a result of the thin multilayer films were observed. Using mica as a substrate, modulations because of the unavoidable internal delaminations in the mica structure also appeared. The resulting oscillations are superpositioned on the transmission features shown on (a) for the same system deposited onto quartz.
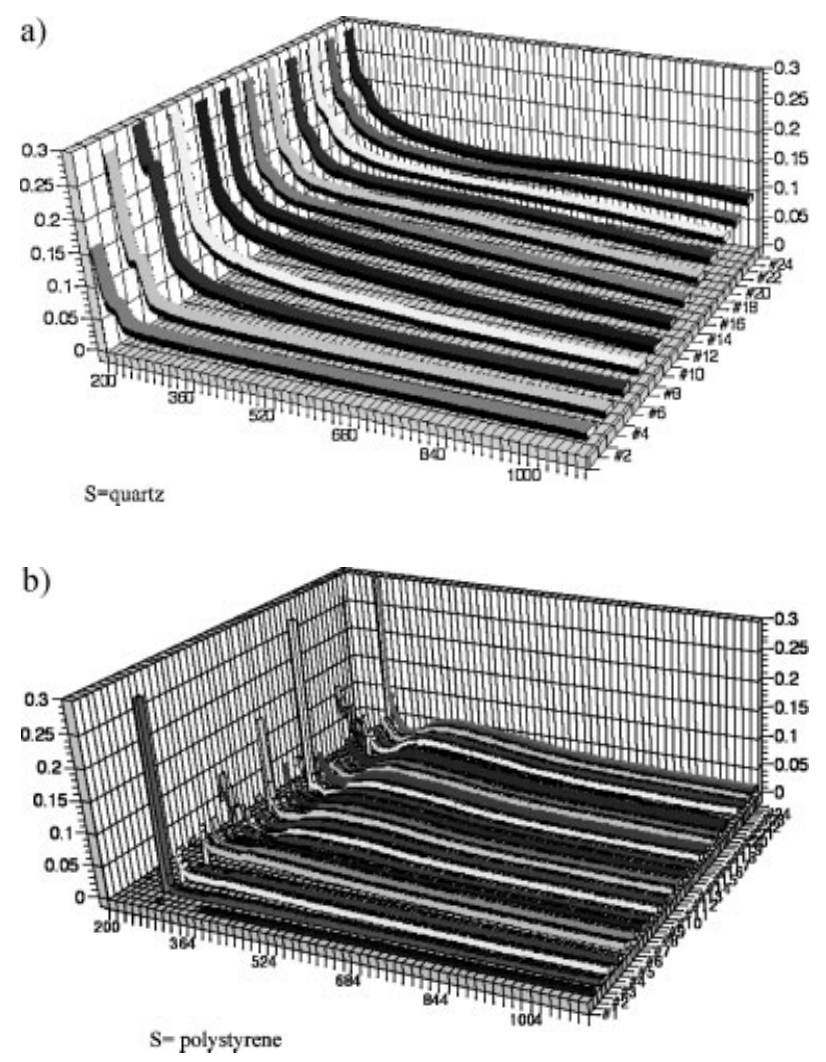

Figure 9. Consecutive spectra of $\left\{\mathrm{Ag}_{66} \mathrm{E} 6\right\} / \mathrm{PSS}$ multilayers on quartz and polystyrene. This PS sample was used for TEM specimen preparation.

even though the resulting spectra still contain the SP peaks and growth of the layers can be confirmed (Figure 9).

\section{Transmission Electron Microscopy}

TEM images of multilayer cross-sections were taken to visualize the nanostructure utilizing the presence of the deposited silver. In Figure 10, dark lines correspond to the silver domains localized in the nanocomposite layers. The average thickness of the dark silver-rich lines corresponds to approximately $25 \mathrm{~nm}$ each. A similar thickness can be observed for PSS. The total thickness of the structure was found to be $d=650-700 \mathrm{~nm}$ because of slight irregularities, although some damage to the multilayer structure is observed on the side of the free surface as a result of microtoming.

\section{General Discussion}

Despite the fact that the specific absorbance of the dendrimer, silver-dendrimer complex, and nanocomposite multilayers are different, the comparative trends in their absorbance spectra carries useful information, because linear transformations of absorbance values into thickness 


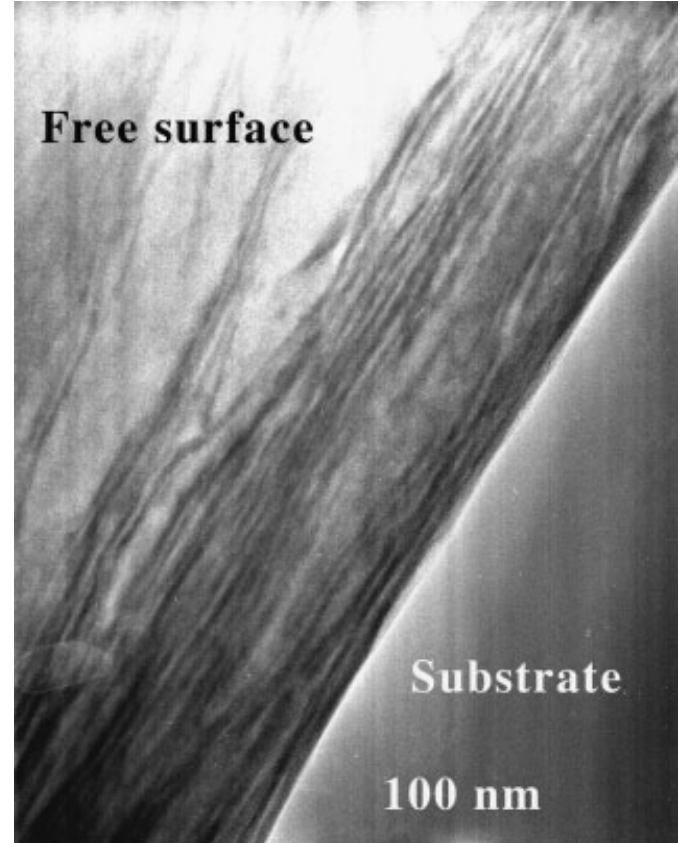

Figure 10. Cross-section image of the $\left(\left\{\mathrm{Ag}_{66} \mathrm{E} 6\right\} / \mathrm{PSS}\right)_{24}$ multilayer deposited on a polystyrene substrate by TEM. Dark lines correspond to the silver domains localized in the nanocomposite layers. The top of the structure has been slightly damaged probably during microtoming.

values will not change the order of the curves. In addition, calculated thickness values (expressed as equivalent PSS thickness) can provide a reasonable minimum value for the multilayers if the complexity is taken into account.

It is possible to estimate layer thicknesses for E5/PSS multilayers by applying the experimentally determined calibration constants of PSS. In polystyrene the $\lambda=254 \mathrm{~nm}$ absorption is the most intense of five peaks that correspond to the $n \rightarrow \pi^{*}$ transitions in the aromatic ring. Consequently, a stepwise increase of total layer thickness at $254 \mathrm{~nm}$ should reflect the amount of deposited PSS when only dendrimer is used as a polycation. Nanocomposites that contain separated metal domains also display absorption in the 290-300 $\mathrm{nm}$ region (depending on template size). ${ }^{[35,43]}$ Therefore Peak $B^{*}$, in those spectra where silver is present (i.e., nanocomposite multilayers), is the cumulative sum of molecular absorptions of polystyrene and Peak S (single nanocomposite domain) peaks.

Thus, calibrations of total sample thickness were possible, based on the measured optical densities both at 224 and $254 \mathrm{~nm}$, i.e., on absorption peaks that correspond to dendrimer + polystyrene and polystyrene absorption resonances, respectively. However, the approx. $50 \mathrm{~nm}$ calculated total thickness is much less than that observed by TEM, which indicates the presence of a considerable amount of water in the multilayers.
Comparing the increase of the absorption responses measured at $\lambda=224 \mathrm{~nm}$ (Figure 11, 12, and 13), the following conclusions can be drawn:

The dendrimer deposition process appears to be different from that of the nanocomposite (Figure 11(a)). The initial regime of the E5 deposition curve has a quadratic form, while growth curves of nanocomposite/PSS layers follow a cubic function. (Note: We were unable to observe the full growth curve for $\mathrm{E} 5$ because of the too high absorption of the total layer over 18 bilayers.)

The layer thickness shows the quickest growth in the case of pure dendrimer $\left(\mathrm{E} 5 . \mathrm{NH}_{2}\right)$ starting with a slow regime, and accelerating with the increasing number of layers (assuming identical dendrimer/PSS deposition ratios as electroneutrality in the top layer must be maintained).

The rate of deposition decreases with increasing silver content even when the $\mathrm{Ag} / \mathrm{N}$ ratio is constant.

Comparison of the growth rates observed for $\left\{\mathrm{Ag}_{17} \mathrm{E} 5\right\} /$ PSS, $\left\{\mathrm{Ag}_{33} \mathrm{E} 5\right\} / \mathrm{PSS}$, and $\left\{\mathrm{Ag}_{66} \mathrm{E} 5\right\} / \mathrm{PSS}$ bilayers at $\lambda=254 \mathrm{~nm}$ as expressed in equivalent dry PSS (Figure 11(b)), reveals an increasing amount of deposited silver with increasing silver content of the nanocomposites (shown by the contribution of $\mathrm{S}$ peaks in Peak $\mathrm{B}^{*}$ ). However, the rate
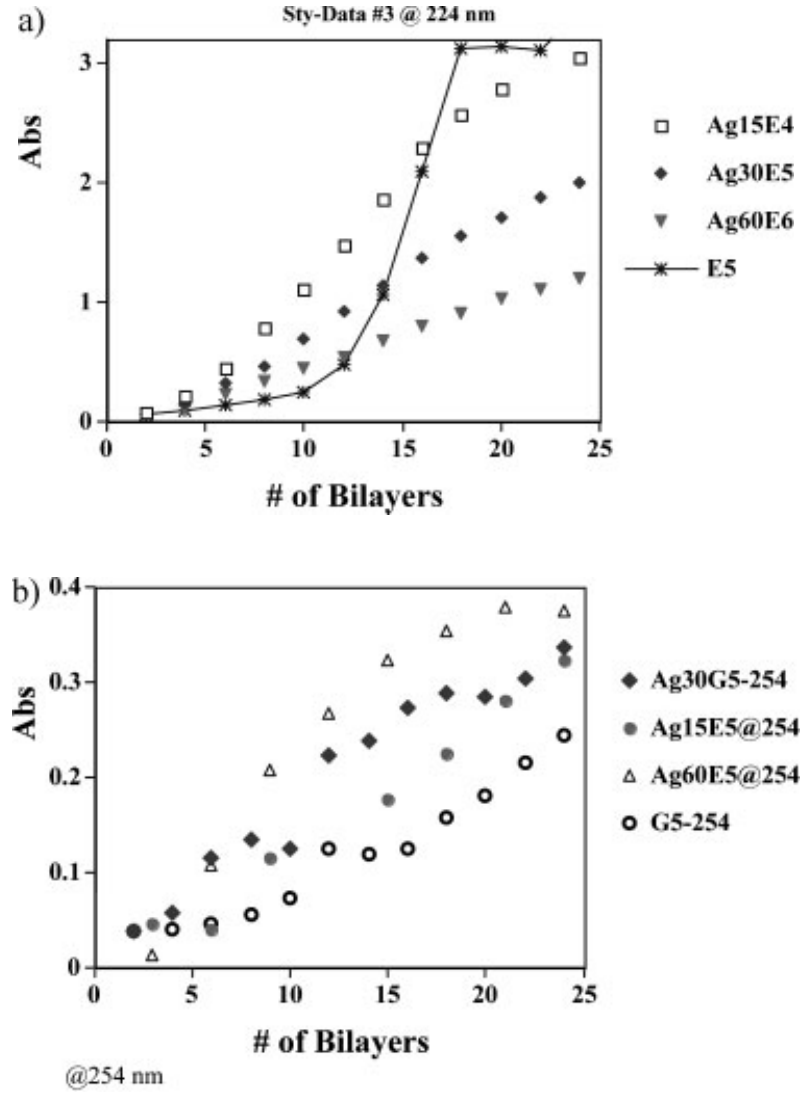

Figure 11. Comparison of the growth rates observed for $E_{5} . A$ dendrimer, $\left\{\mathrm{Ag}_{17} \mathrm{E}_{5}\right\} / \mathrm{PSS}(\mathrm{a}),\left\{\mathrm{Ag}_{33} \mathrm{E}_{5}\right\} / \mathrm{PSS}(\mathrm{b})$, and $\left\{\mathrm{Ag}_{66} \mathrm{E}_{5}\right\} / \mathrm{PSS}$ (c) multilayers at $\lambda=224$ and $254 \mathrm{~nm}$. 


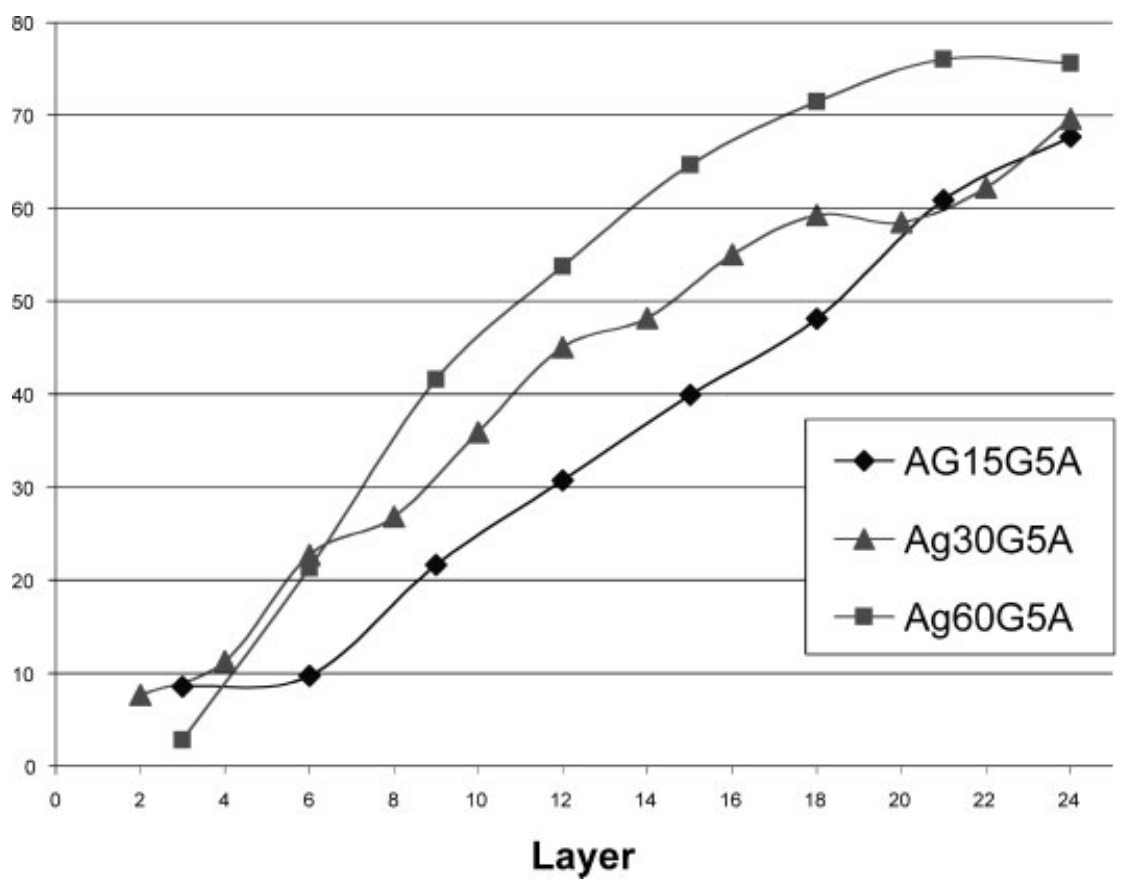

Figure 12. Comparison of the thickness growth rates observed for $\left\{\mathrm{Ag}_{17} \mathrm{E} 5\right\} / \mathrm{PSS},\left\{\mathrm{Ag}_{33} \mathrm{E}_{5}\right\}$ / PSS, and $\left\{\mathrm{Ag}_{66} \mathrm{E}_{5}\right\} / \mathrm{PSS}$ multilayers at $\lambda=224 \mathrm{~nm}$. (Absorption values have been expressed in thickness of equivalent PSS layers.).

increase is less than twofold, which also suggests the deposition of thinner layers when more silver is present.

According to these data, different samples seem to have different cumulative layer thicknesses. Because the electrostatic deposition was carried out for the same length of time for all the samples, therefore, different amounts of charge per nanoparticle must be responsible for the different thicknesses. This would imply the charge is smaller for nanoparticles that contain more silver.

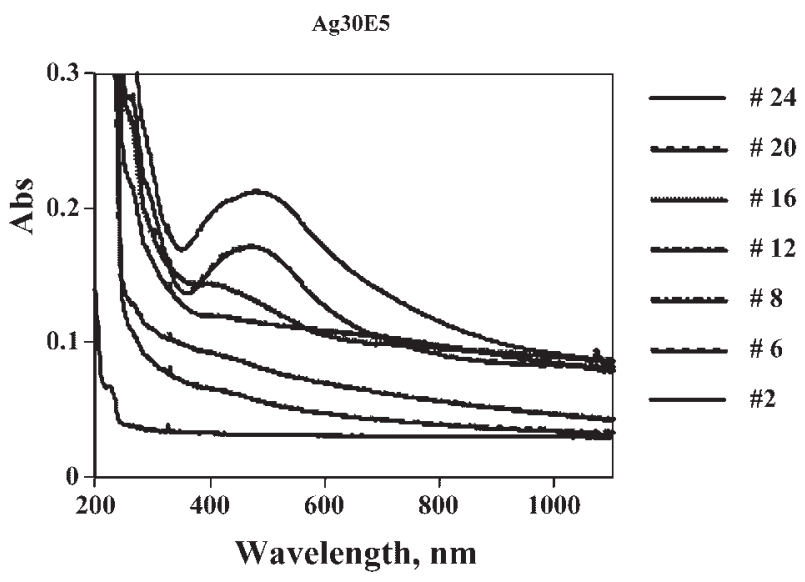

Figure 13. Formation, growth, and shift of SP peak(s) in consecutive spectra of $\left\{\mathrm{Ag}_{33} \mathrm{E} 5\right\} /$ PSS multilayers.
Silver in the hybrid nanoparticles may be present either in ionic or in a zerovalent form. Importantly, because of the equilibrium nature of the complexes, silver content is exchangeable among the dendrimer molecules while $\mathrm{Ag}$ is in the form of an ionic complex. Because of the tendency of the internal metal atoms to collapse into the internal cavities of the dendrimer, ${ }^{[48]}$ only very slow diffusion is possible in the zerovalent form. Because of strong attraction between metal atoms, small clusters will gradually form larger ones, which become increasingly less mobile in the polymer matrix. ${ }^{[55]}$

Metal nanocomposites display several possible SP absorptions depending on the size and composition of the metal nanoparticles. SP peaks have already been observed for gold nanocomposites $^{[39]}$ both in solution and in multilayers, where their wavelength and intensity is also influenced by secondary aggregations. ${ }^{[4]}$

In Figure 13, an SP peak first appears at around $420 \mathrm{~nm}$ (just as in solution), it then shifts towards longer wavelengths (peak maximum is at $500 \mathrm{~nm}$ after 24 bilayers in the case of the ( $\left.\left\{\mathrm{Ag}_{33} \mathrm{E} 5\right\}\right)$ PSS)). The red shift of the SP peak is obviously a result of the increased molecular interactions in the multilayers as compared to the solution state. Although the red-shift of the peak positions suggests increasing interactions between the metallic domains, ${ }^{[4]}$ this red-shift cannot be fully explained by increased aggregations in the individual nanocomposite layers isolated by the PSS layers. Notice that the shape of the absorption envelope also changes, which cannot happen if it was only a concentration effect. Therefore, it is assumed that some interaction does exist between the nanocomposite layers, even though they are separated by hydrophobic PSS layers. Detailed discussion of SP resonances is beyond the scope of this study and any further comment regarding this matter would require a much more complex characterization.

\section{Modulation Features}

Samples with more than the critical number of bilayers showed sizeable regular modulation features in transmission. These modulations arise from multiple reflections within the layered structure, the reflections of which depend on the individual refractive indices of the components used to build the nanometer-scale thin film $\left(n_{i}=\right.$ 1.59-1.60 for polystyrene). ${ }^{[56]}$ Because of the significant differences in refractive indices between the substrate, 
polystyrene, dendrimer, and silver nanocomposite components relative to one another, and to air, modulations are readily observed.

No modulations related to the thickness of the substrate were seen in any tests, as a result of the short coherence length of the spectrophotometer light source. Only reflections within the multilayer structure contributed to the modulated oscillations in optical transmission.

Results for a series of transmission scans are shown in Figure 14(a)-(c), where each scan corresponds to a certain number of deposited multilayers, consequently to samples with different overall thickness.

Films with total thicknesses greater than $\lambda / 4$, capable of exhibiting interference effects, developed progressively greater numbers of smooth oscillations versus wavelength as the number of layers increased, as shown in
Figure 14(a)-(c). The oscillations themselves became modulated when the total thickness of the multilayer on one side of the substrate exceeded $\lambda / 2$. (The increasing oscillation amplitude in Figure 14(c) corresponds to a quarter period of the envelope modulation and Figure 14(a) to three halfperiods.) This 'envelope' modulation constitutes evidence of a dependence on more than one thickness within the layered structure, which confirms the utility of the model.

The films were modeled as a stack of thin dielectric layers of alternating indices of refraction. A characteristic matrix approach was used to solve for the reflectivity and transmission of multiple layers of dielectric materials. The matrix approach allows one to keep track of the multiple reflections and transmissions of the light rays. Keeping track of all the rays for even a few interfaces algebraically is potentially very complicated. With the use of

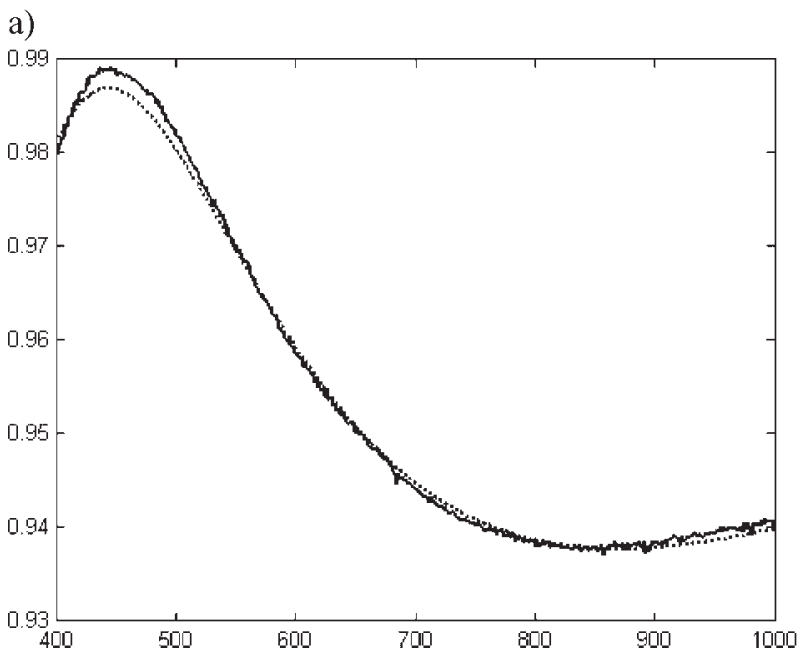

b)

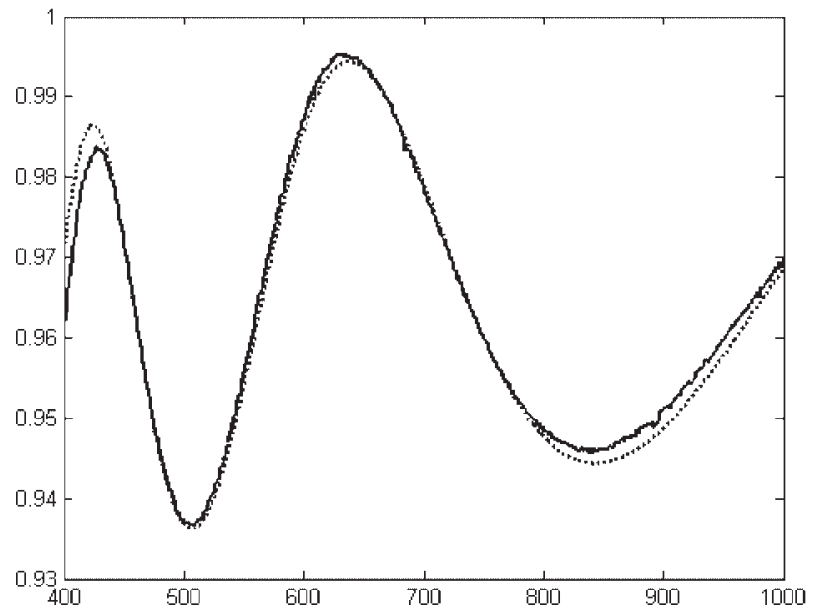

c)

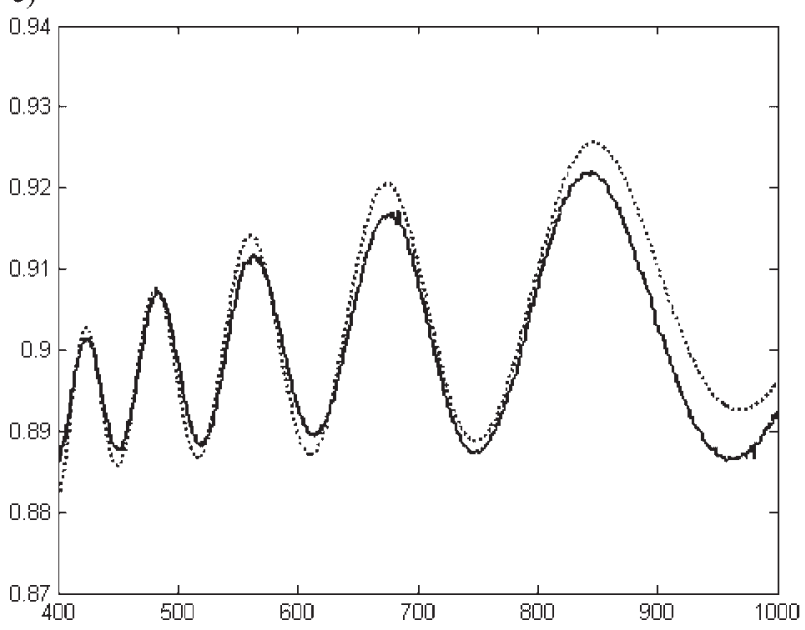

Figure 14. Transmission spectra of multilayer films consisting of: $a)$ an ( $\left.\mathrm{Ag}_{17} \mathrm{E}_{5} / \mathrm{PSS}\right)_{6}$ film, six bilayers on each side of a quartz substrate, $\left.b\right)$ $\left(\mathrm{Ag}_{17} \mathrm{E}_{5} / \mathrm{PSS}\right)_{9}$ film with nine bilayers on each side, and c) a dendrimer-PSS multilayer ( $\left.\mathrm{E}_{5} / \mathrm{PSS}\right)_{24}$ film with 24 bilayers on each side of a quartz substrate. The dotted curves are the theoretical fits and the solid lines are the experimental data. 
matrices, much of the complexity is removed because each characteristic matrix takes into account the effects of the previous layers. The characteristic matrix and its solution for thin layers are given in the appendix.

Simulations were run to fit the data using least-squares fitting. With the limited signal-to-noise ratios achievable in the current apparatus, the individual layer thicknesses are too thin to introduce noticeable modulations in the data. However, combined thicknesses greater than or comparable to a quarter wavelength give measurable modulations. This permitted the entire layered structure to be accurately modeled with a similar approach that replaced the actual heterostructure with only two effective layers whose indices of refraction and thickness were treated as free parameters in fitting the data. This simple model achieved fits as accurate as the full matrix approach, as discussed below. Because of the strong dependence of the modulation patterns on absolute and relative refractive indices and thicknesses of the film components, a reliable fitting procedure was possible for films greater than a quarter wavelength in thickness. As evidenced by the excellence of the theoretical fits to the actual data in Figure 14, optical behavior of samples in this category was dominated by two optically relevant thicknesses, namely the total multilayer thickness and the thickness by the final exterior layer. Envelope modulation requires that the effective index of the initial layers and the final layers be different, as without such a difference no reflection would occur from any internal interface within the multilayer structure at all. Moreover, on the basis of effective medium theory, ${ }^{[57]}$ which dictates that the effective index of a stratified medium is the average of the component indices weighted by their thicknesses, this can only occur if the effective optical thickness or composition of one component of each bilayer increases disproportionately as more layers are added.

The experimental data in Figure $14(\mathrm{~b})$ for the $\left(\mathrm{Ag}_{17} \mathrm{E} 5 /\right.$ PSS) ${ }_{6}$ film can be accurately reproduced when the effective refractive index and thickness for the entire multilayer structure is taken to be different from the effective index and thickness of the final layer. The multiple interference pattern of the double structure model is then computed by taking three interfaces into account, namely the two exterior surfaces and one internal surface. In this case, the indices determined by the two-layer model were $n_{1}=1.551 \pm 0.005$ and $n_{2}=1.555 \pm 0.005$ and the thicknesses were $t_{1}=142 \pm 5 \mathrm{~nm}$ and $t_{2}=267 \pm 4 \mathrm{~nm}$ for a total film thickness of $409 \pm 7 \mathrm{~nm}$. In the case of Figure 14(a) where the film is too thin to exhibit envelope modulation, a single layer model yields the results $n_{1}=1.551 \pm 0.005$ and $t_{1}=142 \pm 5 \mathrm{~nm}$. The value of the imaginary refractive index, which accounted for a small decrease in transmission at short wavelengths was taken to be $\lambda=$ $0.0018 \mathrm{~nm}^{-1}$. Implicitly, abrupt, uniform interfaces are required in order to observe high contrast, regular modulation at all. This establishes the additional important result that individual dendrimer and polystyrene layers as well as DNC layers and polystyrene layers have negligible interpenetration, they are flat and uniform.

We have tested the validity of our results by applying the same model to a well-known regularly layered material structure such as mica. In real mica structures strong internal reflections arise from several occasional laminations at fractional wave thicknesses (Figure 15(a)). In Figure 15(a), a more complex simulated modulation pattern is shown, which is expected for slightly thicker but otherwise similar structures to those in Figure 14(b)
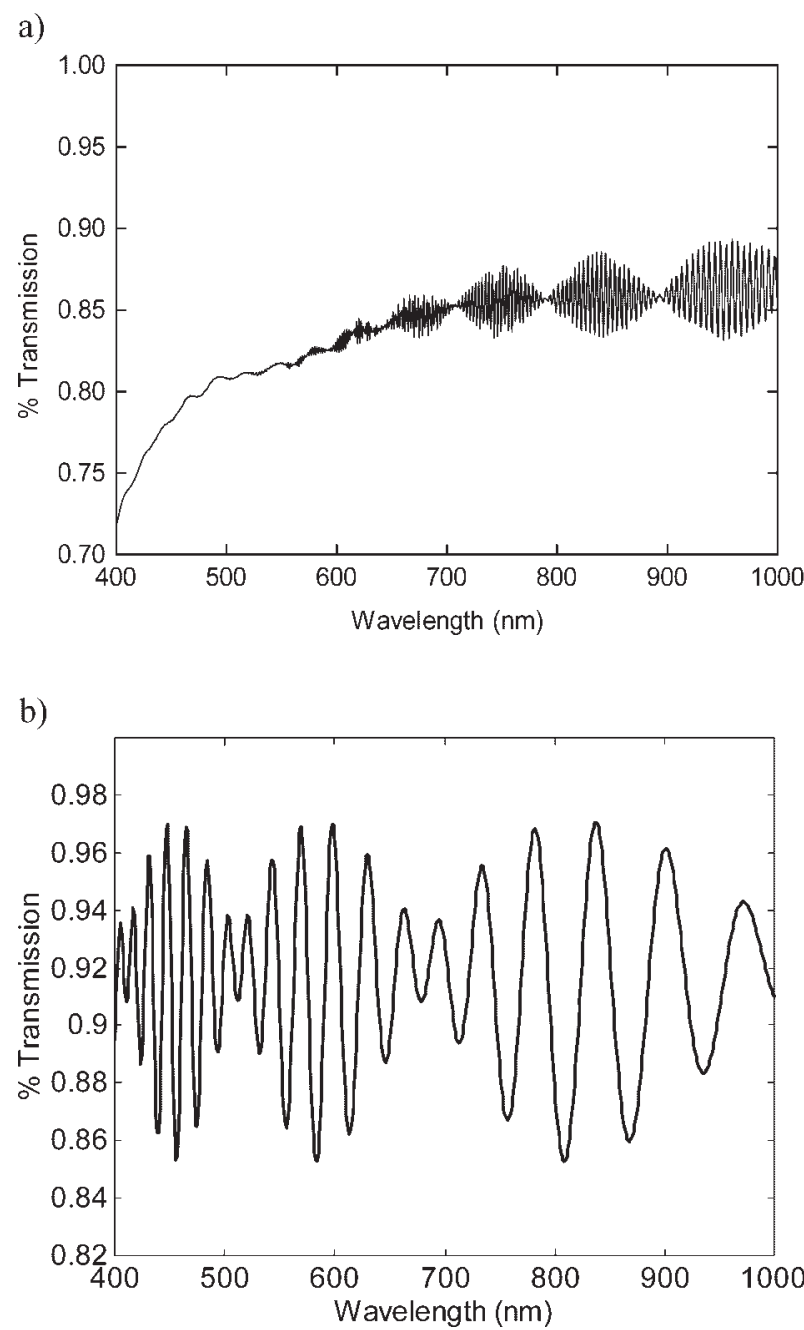

Figure 15. a) Simulation of transmission through a two-layer structure. Thickness of the two layers were taken to be $t_{1}=640 \mathrm{~nm}$ and $t_{2}=3500 \mathrm{~nm}$ and their indices were 1.6 and 1.5, respectively. b) Experimental trace of the transmission of a mica substrate showing complex modulations arising from a partially delaminated structure. 
and 14(c). Clearly, this is the mechanism responsible for the complex patterns observed.

In summary, it is suggested that the observed spectra of the nanocomposite/PSS multilayers (without the substrate) is composed of:

$$
A=A_{\mathrm{el}}+A_{\mathrm{DNC}}+A_{\mathrm{IL}}+A_{\mathrm{LL}}
$$

Where $A$ is the total absorption curve, $A_{\mathrm{el}}$ is the electron excitation spectra from the organic molecule, $A_{\mathrm{DNC}}$ is the single nanocomposite primary spectrum (particle surface plasmon resonances, $\left.\mathrm{SP}_{1}\right), A_{\mathrm{IL}}$ is the interlayer resonances as a result of interacting domains within the DNC layers surface $\left(\mathrm{SP}_{\mathrm{IL}}\right.$ ), and $A_{\mathrm{LL}}$ is the resonance attributed to interactions between metal nanoparticle-containing layers ( $\left.\mathrm{SP}_{\mathrm{LL}}\right)$.

The actual absorption/transmission spectrum, therefore, depends both on the composition and the layer structure and involves contributions from interparticle, intralayer, and interlayer interactions.

The structure of the resulting DNC multilayers is depicted on Figure 16. DNC layers are more distinctly separated than linear polymers. Moreover, the high water content of the dendrimer-containing layers allows for diffusion of silver still in ionic form. No silver atoms/domains/particles are found in the more hydrophobic PSS layers. Increasing the silver concentration in the starting complexes results in the deposition of thinner bilayers with higher metal content. This feature allows us to manipulate the structure of the nanocomposite multilayer films.

a)

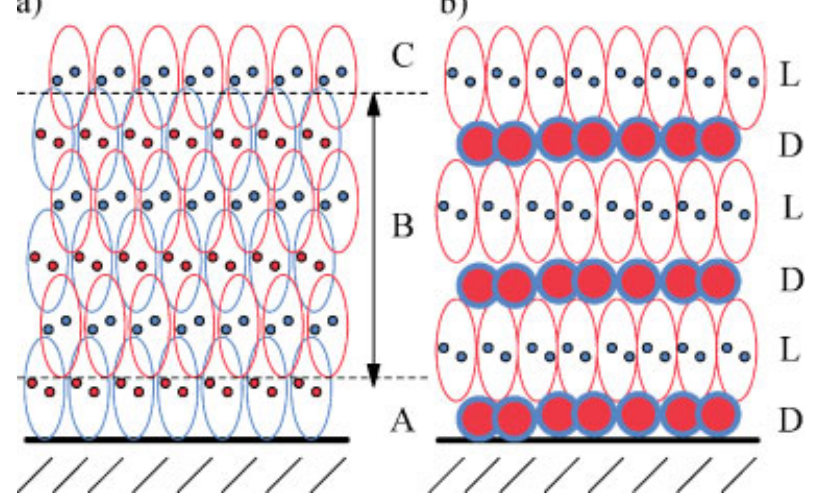

Figure 16. Schematic comparison of multilayer structures. (a): Multilayers built from identical linear polycations and linear polyanions (both carrying small counterions). The oblate shape is to depict the envelope of overlapping polymer coils and to illustrate partial interpenetration. $\mathrm{A}=$ Zone $\mathrm{I}, \mathrm{B}=$ Zone II, and $\mathrm{C}=$ Zone III. (b): Multilayer structure built from DNC polycations and linear polyanions. Use of impenetrable DNC nanoparticles results in a different kind of highly defined multilayer structure, in which local properties are much better defined. $\mathrm{L}=$ linear polymer layer, $\mathrm{D}=\mathrm{DNC}$ layer.

\section{Conclusion}

The characterization and optical properties of ultrathin DNC multilayers that contain nanosized metallic silver domains in well-structured organic thin films is described. Fabrication was achieved using layer-by-layer electrostatic assembly techniques on various substrates. Deposited silver-poly(amidoamine) complex and nanocomposite ultrathin layers were separated by thin layers of sodium poly(styrene sulfonate). Actual UV-vis spectra of the multilayers were found to be the combination of absorptions from electronic transitions, surface plasmon peaks, and high contrast, regular frequency modulations as a result of the film structure. Use of DNCs in multilayers resulted in abrupt, flat, and uniform interfaces, in which DNC and poly(styrene sulfonate) layers had negligible interpenetration. Modulations in UV-vis absorption appeared as a consequence of the regular multilayer growth. Absorption curves were found to be the function of composition, particle size, number of deposited layers, and the multilayer structure. A simple optical model was applied, which permitted the entire layered structure to be accurately modeled by only two effective layers whose indices of refraction and thickness were treated as free parameters. The combination of an electrostatic multilayer assembly technique with DNC materials offers highly uniform multilayers, controlled layer thicknesses, and $z$ directional composition control as well as an atom-byatom control in the individual nanoparticles.

Acknowledgements: This work was supported in part by the MRSEC Program of the National Science Foundation under Award Number DMR-9809687\#2. S.C.R. gratefully acknowledges partial grant support from the National Science Foundation (DMR9975542) and U.S. DOD Air Force (F49620-99-1-0158).

Received: May 7, 2007; Accepted: May 15, 2007; DOI: 10.1002/ mabi.200700114

Keywords: dendrimers; nanocomposites; nanolayers; structureproperty relations; UV-vis spectroscopy

\section{Appendix}

The characteristic matrix of a single layer is

$$
m_{j}=\left[\begin{array}{cc}
\cos \left(2 \pi n_{j} h_{j} / \lambda\right) & i \sin \left(2 \pi n_{j} h_{j} / \lambda\right) / n_{j} \\
i \sin \left(2 \pi n_{j} h_{j} / \lambda\right) n_{j} & \cos \left(2 \pi n_{j} h_{j} / \lambda\right)
\end{array}\right]
$$

where $n_{j}$ is the index of refraction of the $j$ th layer and $h_{j}$ is the thickness of the $j$ th layer. The overall matrix can then be found by multiplying the characteristic matrices of successive layers:

$$
M=m_{1} m_{2} \ldots m_{n},
$$


with careful attention to the order in which they are applied. $^{[58]}$

The reflectivity and transmission are then calculated as:

$$
R=|r|^{2}
$$

where $r=\left(n_{\mathrm{o}} B-C\right) /\left(n_{\mathrm{o}} B+C\right)$, and

$$
T=n_{\text {sub }}|t|^{2} / n_{\mathrm{o}}
$$

where $t=2 n_{\mathrm{o}} /\left(n_{\mathrm{o}} B+C\right)$

The values of $B$ and $C$ are found from

$$
\left[\begin{array}{l}
B \\
C
\end{array}\right]=M\left[\begin{array}{c}
1 \\
n_{\text {sub }}
\end{array}\right]
$$

where $n_{\mathrm{o}}$ is the index of refraction of incident material and $n_{\text {sub }}$ is the index of refraction of substrate.

If the layers are periodic, then there is an analytic solution ${ }^{[59]}$ for the overall matrix with the individual elements of $M$ being given by $M_{i, j}$ where

$$
\begin{aligned}
M_{11}= & {\left[\cos \left(\beta_{2}\right) \cos \left(\beta_{3}\right)\right.} \\
& \left.-\left(p_{3} / p_{2}\right) \sin \left(\beta_{2}\right) \sin \left(\beta_{3}\right)\right] U_{N-1}(a)-U_{N-2}(a) \\
M_{12}= & -i\left[\left(1 / p_{3}\right) \cos \left(\beta_{2}\right) \sin \left(\beta_{3}\right)\right. \\
& \left.+\left(1 / p_{2}\right) \sin \left(\beta_{2}\right) \cos \left(\beta_{3}\right)\right] U_{N-1}(a) \\
M_{21}= & -i\left[p_{2} \sin \left(\beta_{2}\right) \cos \left(\beta_{3}\right)\right. \\
& \left.+p_{3} \cos \left(\beta_{2}\right) \sin \left(\beta_{3}\right)\right] U_{N-1}(a) \\
& \left.-\left(p_{2} / p_{3}\right) \sin \left(\beta_{2}\right) \sin \left(\beta_{3}\right)\right] U_{N-1}(a)-U_{N-2}(a) .
\end{aligned}
$$

Here $a=\cos \left(\beta_{2}\right) \cos \left(\beta_{3}\right)-(1 / 2)\left(p_{2} / p_{3}+p_{3} / p_{2}\right) \sin \left(\beta_{2}\right)$ $\sin \left(\beta_{3}\right), N$ is the number of bilayers, $U$ is a Chebyshev polynomial of the second kind, $p_{i}=n_{i}$, and $\beta_{i}=(2 \pi / \lambda) n_{i} h_{i}$.

These equations assume normally incident light. If the light is not incident normal to the layers, at normal incidence, then the equations must be modified slightly.

[1] S. Ayappan, R. S. Gopalan, G. N. Subbana, C. N. R. Rao, J. Mater. Res. 1997, 12, 398.

[2] K. Puech, W. Blau, A. Grund, C. Bubeck, G. Cardenas, Opt. Lett. 1995, 20, 1613

[3] G. Decher, J. D. Hong, J. Schmitt, Thin Solid Films 1992, 210, 831.

[4] G. Decher, Science 1997, 277, 1232.

[5] W. Knoll, Curr. Opin. Colloid Interface Sci. 1996, 1, 137.

[6] A. Laschewsky, Eur. Chem. Chronicle 1997, 2, 13.

[7] G. Decher, "The Polymeric Materials Encyclopedia: Synthesis, Properties, and Applications", J. C. Salamone, Ed., CRC Press, Boca Raton, FL 1996, Vol. 6, p. 4540.
[8] H. Dautzenberg, J. Hartmann, S. Grunewald, F. Brand, Ber. Bunsenges. Phys. Chem. 1996, 100, 1024

[9] W. Chen, T. J. McCarthy, Macromolecules 1997, 30, 78.

[10] G. Ladam, P. Schaad, J. C. Voegel, P. Schaaf, G. Decher F. Cuisinier, Langmuir 2000, 16, 1249-1255.

[11] Y. Lvov, G. Decher, H. Mohwald, Langmuir 1993, 9, 481

[12] M. Losche, J. Schmitt, G. Decher, W. G. Bouwman, K. Kjær Macromolecules 1998, 31, 8893.

[13] D. A. Tomalia, J. R. Dewald, M. J. Hall, S. J. Martin, P. B. Smith First SPSJ Int. Polym. Conference, Kyoto, Japan, August 1984, p. 65.

[14] D. A. Tomalia, H. Baker, J. Dewald, M. Hall, M. Kallos, S. Martin, J. Roeck, J. Ryder, P. Smith, Polym. J. (Tokyo) 1985, 17, 117.

[15] G. R. Newkome, Z.-O. Yao, G. R. Baker, V. K. Gupta, J. Org. Chem. 1985, 50, 2003.

[16] C. J. Hawker, J. M. J. Frechet, J. Am. Chem. Soc. 1990, 112 7638.

[17] D. A. Tomalia, A. M. Naylor, W. A. Goddard, III, Angew. Chem. Int. Ed. 1990, 29, 138.

[18] P. G. de Gennes, H. Hervet, J. de Physique Lett. (Paris) 1983 44, 9, 351.

[19] M. L. Mansfield, L. I. Klushin, Macromolecules 1993, 26 , 4262.

[20] M. K. Bhalgat, J. C. Roberts, Eur. Polym. J. 2000, 36, 647.

[21] S. A. Evenson, J. P. Badyal, Adv. Mater. 1997, 9, 1097.

[22] V. V. Tsukruk, F. Rinderspacher, V. N. Bliznyuk, Langmuir 1997, 13, 2171.

[23] M. L. Mansfield, Polymer 1996, 37, 3835

[24] V. V. Tsukruk, Adv. Mater. 1998, 10, 253.

[25] V. N. Bliznyuk, F. Rinderspacher, V. V. Tsukruk, Polymer 1998 39, 5249.

[26] K. Esumi, M. Goino, Langmuir 1998, 14, 4466.

[27] S. Watanabe, S. L. Regen, J. Am. Chem. Soc. 1994, 116, 8855.

[28] J. Khopade, F. Caruso, Langmuir 2002, 18, 7669.

[29] J. Khopade, F. Caruso, Nano Lett. 2002, 2, 415.

[30] L. Balogh, D. R. Swanson, R. Spindler, D. A. Tomalia, "Formation and Characterization of Dendrimer-Based Water Soluble Inorganic Nanocomposites", Proceedings of ACS PMSE 1997, Vol. 77, 118.

[31] N. Beck Tan, L. Balogh, S. Trevino, "Structure of MetalloOrganic Nanocomposites Produced from Dendrimer Complexes", Proceedings of ACS PMSE 1997, Vol. 77, 120.

[32] L. Balogh, D. A. Tomalia, G. L. Hagnauer, Chemical Innovation, March, 2000, p. 19.

[33] US 6995234 (2006), invs.: D. A. Tomalia, L. Balogh.

[34] US 6664315 B2 (2003), invs.: D. A. Tomalia, L. Balogh.

[35] L. Balogh, D. A. Tomalia, J. Am. Chem. Soc. 1998, 120, 7355.

[36] M. Zhao, L. Sun, R. M. Crooks, J. Am. Chem. Soc. 1998, 120 4877.

[37] K. Sooklal, L. H. Hanus, H. J. Ploehn, C. J. Murphy, Adv. Mater 1998, 10, 1083.

[38] K. Esumi, A. Suzuki, N. Aihara, K. Usui, K. Torigoe, Langmuir 1998, 14, 3157.

[39] J.-A. He, R. Valluzzi, K. Yang, T. Dolukhanyan, C. Sung J. Kumar, S. K. Tripathy, L. Samuelson, L. Balogh, D. A. Tomalia, Chem. Mater. 1999, 11, 3268.

[40] Y. J. Lvov, H. Haas, G. Decher, H. Möhwald, A. Kalachev, Phys. Chem. 1993, 97, 12835.

[41] S. Rubin, G. Bar, R. W. Cutts, T. A. Zawodzinski, Jr., Proc. Electrochem. Soc. 1995, 151.

[42] S. Rubin, R. W. Cutts, T. N. Taylor, T. A. Zawodzinski, Jr., Langmuir 1996, 12, 1172. 
[43] L. Balogh, R. Valluzzi, G. L. Hagnauer, K. S. Laverdure, S. P. Gido, D. A. Tomalia, J. Nanoparticle Res. 1999, 1, 353.

[44] M. Zhao, R. M. Crooks, Chem. Mater. 1999, 11, 3379.

[45] N. Beck Tan, L. Balogh, S. Trevino, D. A. Tomalia, J. S. Lin, Mater. Res. Soc. Symp. Proc. 1998, 519, 143.

[46] N. Beck Tan, L. Balogh, S. Trevino, D. A. Tomalia, J. S. Lin, Polymer 1999, 40, 2537.

[47] L. Balogh, K. S. Laverdure, S. P. Gido, A. G. Mott, M. J. Miller, B. P. Ketchel, D. A. Tomalia, Mater. Res. Soc. Symp. Proc. 1999, $576,69$.

[48] M. F. Ottaviani, R. Valluzzi, L. Balogh, Macromolecules 2002, 35, 5105.

[49] W. Lesniak, A. U. Bielinska, K. Sun, K. W. Janczak, X. Shi, J. R. Baker, Jr., L. P. Balogh, Nano Lett. 2005, 5, 2123.

[50] S. M. Milas, J. Y. Ye, T. B. Norris, L. P. Balogh, J. R. Baker, Jr, K. W. Hollman, S. Emelianov, M. O’Donnell, Appl. Phys. Lett. 2003, 82, 994
[51] R. G. Ispasoiu, L. Balogh, O. P. Varnavski, D. A. Tomalia, T. Goodson, III, J. Am. Chem. Soc. 2000, 122, 11005.

[52] J. Y. Ye, L. Balogh, T. B. Norris, Appl. Phys. Lett. 2002, 80, 1713.

[53] S. M. Milas, J. Y. Ye, T. B. Norris, L. P. Balogh, J. R. Baker, Jr., K. W. Hollman, S. Emelianov, M. O'Donnell, Appl. Phys. Lett. 2003, 82, 994.

[54] L. Balogh, D. R. Swanson, D. A. Tomalia, G. L. Hagnauer, A. T. McManus, Nano Lett. 2001, 1, 18.

[55] F. Gröhn, B. J. Bauer, Y. A. Akpalu, C. L. Jackson, E. J. Amis, Macromolecules 2000, 33, 6042.

[56] "Polymer Handbook", J. Brandrup, E. H. Immergut, E. A. Grulke, Eds., $4^{\text {th }}$ edition, Wiley Interscience, New York 1999.

[57] J. C. Maxwell-Garnett, Trans. R. Soc. London 1904, 203, 385.

[58] W. H. Southwell, Vacuum and Thin Film 1999, 2, 17.

[59] M. Born, E. Wolf, "Principles of Optics", $6^{\text {th }}$ edition, Pergamon Press. London: reprinted 1993. 